SLAC-PUB-12804

September 2007

\title{
AdS/CFT and Exclusive Processes in QCD
}

\author{
Stanley J. Brodsky $\left.\right|^{\circledast 2}$ and Guy F. de Téramond $\rrbracket^{\oplus, a}$ \\ ${ }^{a}$ Stanford Linear Accelerator Center, Stanford University, Stanford, CA 94309, USA \\ ${ }^{b}$ Universidad de Costa Rica, San José, Costa Rica
}

\begin{abstract}
The AdS/CFT correspondence between string theory in AdS space and conformal field theories in physical space-time leads to an analytic, semi-classical model for strongly-coupled QCD which has scale invariance and dimensional counting at short distances and color confinement at large distances. One can use holography to map the amplitude describing the hadronic state in the fifth dimension of Anti-de Sitter space $\mathrm{AdS}_{5}$ to the light-front wavefunctions of hadrons in physical space-time, thus providing a relativistic description of hadrons in QCD at the amplitude level. In particular, we show that there is an exact correspondence between the fifth-dimensional coordinate of AdS space $z$ and a specific impact variable $\zeta$ which measures the separation of the quark and gluonic constituents within the hadron in ordinary space-time. New relativistic light-front equations in ordinary space-time can then be derived which reproduce the results obtained using the 5-dimensional theory. The effective light-front equations possess elegant algebraic structures and integrability properties. This connection between the AdS and the light-front representations allows one to compute the analytic form of the frame-independent light-front wavefunctions, the fundamental entities which encode hadron properties and allow the computation of decay constants, form factors, deeply virtual Compton scattering, exclusive heavy hadron decays and other exclusive scattering amplitudes. As specific examples we compute the pion coupling constant $f_{\pi}$ and study the behavior of the pion form factor $F_{\pi}\left(q^{2}\right)$ in the space and time-like regions. We also determine the Dirac nucleon form factors $F_{1}^{p}\left(q^{2}\right)$ and $F_{1}^{n}\left(q^{2}\right)$ in the space-like region.
\end{abstract}

Invited talk presented at

Workshop on Exclusive Reactions at High Momentum Transfer

21-24 May 2007

Newport News, Virginia

\footnotetext{
*Electronic address: sjbth@slac.stanford.edu

${ }^{\dagger}$ Electronic address: gdt@asterix.crnet.cr
} 


\section{Exclusive Processes in QCD}

Exclusive processes provide essential tests of the quark and gluon structure of hadrons at the amplitude level and a window into the form of bound-state wavefunctions of QCD. For example, hadron form factors and the generalized distributions which control deeply virtual Compton scattering are expressible in terms of the light-front wavefunctions $\psi_{n}^{H}\left(x_{i}, \mathbf{k}_{\perp i}, \lambda_{i}\right)$, the bound-state eigensolutions of the QCD light-front Hamiltonian.

Light-front wavefunctions are the relativistic generalizations of the familiar Schrödinger wavefunctions of atomic physics, but they are determined at fixed light-front time $\tau=t+z / c$ - the "front form" advocated by Dirac [1] - rather than at fixed ordinary time $t$. The light-front wavefunctions of a hadron are independent of the momentum of the hadron, and they are thus boost invariant; Wigner transformations and Melosh rotations are not required. The light-front formalism for gauge theories in light-cone gauge is particularly useful in that there are no ghosts and one has a direct physical interpretation of orbital angular momentum.

Given the light-front wavefunctions $\psi_{n / h}$ one can compute a large range of hadron observables. For example, the valence and sea quark and gluon distributions which are measured in deep inelastic lepton scattering are defined from the squares of the LFWFS summed over all Fock states $n$. Form factors, exclusive weak transition amplitudes [2] such as $B \rightarrow \ell \nu \pi$. and the generalized parton distributions [3] measured in deeply virtual Compton scattering $\gamma^{*} p \rightarrow \gamma p$ are (assuming the "handbag" approximation) overlaps of the initial and final LFWFS with $n=n^{\prime}$ and $n=n^{\prime}+2$. The gauge-invariant distribution amplitude $\phi_{H}\left(x_{i}, Q\right)$ defined from the integral over the transverse momenta $\mathbf{k}_{\perp i}^{2} \leq Q^{2}$ of the valence (smallest $n$ ) Fock state provides a fundamental measure of the hadron at the amplitude level [4, 5]; they are the nonperturbative input to the factorized form of hard exclusive amplitudes and exclusive heavy hadron decays in perturbative QCD. The resulting distributions obey the DGLAP and ERBL evolution equations as a function of the maximal invariant

mass, thus providing a physical factorization scheme [6]. In each case, the derived quantities satisfy the appropriate operator product expansions, sum rules, and evolution equations. At large $x$ where the struck quark is far-off shell, DGLAP evolution is quenched [7], so that the fall-off of the DIS cross sections in $Q^{2}$ satisfies Bloom-Gilman inclusive-exclusive duality at fixed $W^{2}$.

One of the most significant theoretical advances in recent years has been the application of the AdS/CFT correspondence [8] between string theories defined in 5dimensional Anti-de Sitter (AdS) space-time and conformal field theories in physical space-time. QCD is not itself a conformal theory; however there are indications, both 
from theory [9, 10] and phenomenology [11, 12], that the QCD coupling is slowly varying at small momentum transfer. In addition, one can argue that if the gluon has a maximum wavelength or an effective mass [13] due to confinement, that gluonic vacuum polarization corrections and the $\beta$-function must vanish in the infrared.

If there is a conformal window where the QCD coupling is large and approximately constant and quark masses can be neglected, then QCD resembles a conformal theory, thus motivating the application of AdS/CFT to QCD. Thus, even though QCD is not conformally invariant, one can use the mathematical representation of the conformal group in five-dimensional anti-de Sitter space to construct an analytic first approximation to the theory. The resulting AdS/QCD model gives accurate predictions for hadron spectroscopy and a description of the quark structure of mesons and baryons which has scale invariance and dimensional counting at short distances, together with color confinement at large distances.

As we shall discuss, one can use holography to map the amplitude $\Phi(z)$ describing the hadronic state in the fifth dimension of Anti-de Sitter space $\mathrm{AdS}_{5}$ to the lightfront wavefunctions $\psi_{n / h}$ of hadrons in physical space-time [14], thus providing a relativistic description of hadrons in QCD at the amplitude level. In fact, there is an exact correspondence between the fifth-dimensional coordinate of anti-de Sitter space $z$ and a specific impact variable $\zeta$ in the light-front formalism which measures the physical separation of the constituents within the hadron. One can derive this correspondence by noticing that the mapping of $z \rightarrow \zeta$ transforms the expression for the form factors in AdS/CFT to the exact QCD Drell-Yan-West expression in terms of light-front wavefunctions. The amplitude $\Phi(z)$ describing the hadronic state in $\mathrm{AdS}_{5}$ can then be precisely mapped to the light-front wavefunctions $\psi_{n / h}$ of hadrons in physical space-time [14]. One thus obtains not only an accurate description of the hadron spectrum, but also a simple but realistic model of the valence light-front wavefunctions of mesons, baryons, and glueballs. Thus there is a remarkable mapping between the AdS description of hadrons and the Hamiltonian formulation of QCD in physical space-time quantized at fixed light front time $\tau=t+z / c$.

The AdS/QCD correspondence is particularly relevant for the description of hadronic form factors, since it incorporates the connection between the twist of the hadron to the fall-off of its current matrix elements, as well as essential aspects of vector meson dominance. It also provides a convenient framework for analytically continuing the space-like results to the time-like region. Recent applications to the form factors of mesons and nucleons [15, 16, 17, 18, 19, 20, 21] have followed from the pioneering work of refs. [22, 23]. 


\section{The AdS/CFT Duality}

The essential principle underlying the AdS/CFT approach to conformal gauge theories is the isomorphism of the group of Poincare' and conformal transformations $S O(4,2)$ to the group of isometries of Anti-de Sitter space. The AdS metric is

$$
d s^{2}=\frac{R^{2}}{z^{2}}\left(\eta_{\mu \nu} d x^{\mu} d x^{\nu}-d z^{2}\right)
$$

which is invariant under scale changes of the coordinate in the fifth dimension $z \rightarrow \lambda z$ and $x_{\mu} \rightarrow \lambda x_{\mu}$. Thus one can match scale transformations of the theory in $3+1$ physical space-time to scale transformations in the fifth dimension $z$. In the AdS/CFT duality, the amplitude $\Phi(z)$ represents the extension of the hadron into the additional fifth dimension. The behavior of $\Phi(z) \rightarrow z^{\Delta}$ at $z \rightarrow 0$ matches the twist-dimension of the hadron at short distances $x^{2} \rightarrow 0$.

\subsection{The Hard-Wall Holographic Model}

As shown by Polchinski and Strassler [24], one can simulate confinement by imposing boundary conditions in the holographic variable at $z=z_{0}=1 / \Lambda_{\mathrm{QCD}}$. Confinement can also be introduced by modifying the AdS metric to mimic a confining potential. The resulting models, although ad hoc, provide a simple semi-classical approximation to QCD which incorporate both constituent counting rule behavior at short distances and confinement at large distances.

This simple approach, which has been described as a "bottom-up" approach, has been successful in obtaining general properties of scattering amplitudes of hadronic bound states at strong coupling [24, 25], the low-lying hadron spectra [26, 27, 28, 29] and hadron couplings and chiral symmetry breaking [30, 31, 32, 33]. The gauge theory/gravity duality also provides a convenient framework for the description of deep inelastic scattering structure functions at small $x$ [34] and a unified description of hard and soft pomeron physics [35]. Recent applications to describe chiral symmetry breaking [36] and other meson and baryon properties, have also been carried out within the framework of a top-bottom approach to AdS/CFT using higher dimensional branes [37.

\subsection{The Soft-Wall Holographic Model}

The predicted mass spectrum in the truncated space hard-wall (HW) model is linear $M \propto L$ at high orbital angular momentum $L$, in contrast to the quadratic dependence $M^{2} \propto L$ in the usual Regge parameterization. It has been shown recently that by 
choosing a specific profile for a non-constant dilaton, the usual Regge dependence can be obtained [38]. The procedure allows one to retain conformal AdS metrics (1) and to introduce a smooth cutoff which depends on the profile of a dilaton background field $\varphi$

$$
S=\int d^{4} x d z \sqrt{g} e^{-\varphi(z)} \mathcal{L}
$$

where $\varphi$ is a function of the holographic coordinate $z$ which vanishes in the ultraviolet limit $z \rightarrow 0$. The IR hard-wall or truncated space holographic model, discussed in the previous section, corresponds to a constant dilaton field in the confining region $\varphi(z)=\varphi_{0}, z \leq 1 / \Lambda_{\mathrm{QCD}}$, and to very large values elsewhere: $\varphi(z) \rightarrow \infty$ for $z>$ $1 / \Lambda_{\mathrm{QCD}}$. The introduction of a soft cutoff avoids the ambiguities in the choice of boundary conditions at the infrared wall. A convenient choice [38] for the background field with usual Regge behavior is $\varphi(z)=\kappa^{2} z^{2}$. The resulting wave equations are equivalent to the radial equation of a two-dimensional oscillator previously found in the context of mode propagation on $A d S_{5} \times S^{5}$ in the light-cone formulation of Type II supergravity 39.

\section{Light-Front Fock Representation}

The light-front expansion is constructed by quantizing QCD at fixed light-cone time [1 $\tau=t+z / c$ and forming the invariant light-front Hamiltonian: $H_{L F}^{Q C D}=P^{+} P^{-}-\vec{P}_{\perp}^{2}$ where $P^{ \pm}=P^{0} \pm P^{z}$ [40]. The momentum generators $P^{+}$and $\vec{P}_{\perp}$ are kinematical; i.e., they are independent of the interactions. The generator $P^{-}=i \frac{d}{d \tau}$ generates

light-cone time translations, and the eigen-spectrum of the Lorentz scalar $H_{L F}^{Q C D}$ gives the mass spectrum of the color-singlet hadron states in QCD: $H_{L C}\left|\psi_{h}\right\rangle=\mathcal{M}_{h}^{2}\left|\psi_{h}\right\rangle$. The state $\left|\psi_{h}\right\rangle$ is an expansion in multi-particle Fock eigenstates $\{|n\rangle\}$ of the free light-front Hamiltonian: $\left|\psi_{h}\right\rangle=\sum_{n} \psi_{n / h}\left|\psi_{h}\right\rangle$; the projection of the eigensolution on the free Fock basis gives the hadronic light-front wavefunctions. The coefficients of the Fock expansion $\psi_{n / h}\left(x_{i}, \mathbf{k}_{\perp i}, \lambda_{i}\right)$ are independent of the total momentum $P^{+}$and $\mathbf{P}_{\perp}$ of the hadron and depend only on the relative partonic coordinates, the longitudinal momentum fraction $x_{i}=k_{i}^{+} / P^{+}$, the relative transverse momentum $\mathbf{k}_{\perp i}$ and $\lambda_{i}$, the constituent's spin along the $z$ direction. Momentum conservation requires $\sum_{i=1}^{n} x_{i}=1$ and $\sum_{i=1}^{n} \mathbf{k}_{\perp i}=0$.

\subsection{Light-Front Wavefunctions in Impact Space}

The holographic mapping of hadronic LFWFs to AdS string modes is most transparent when one uses the impact parameter space representation [41]. The total position 
coordinate of a hadron or its transverse center of momentum $\mathbf{R}_{\perp}$, is defined in terms of the energy momentum tensor $T^{\mu \nu}$

$$
\mathbf{R}_{\perp}=\frac{1}{P^{+}} \int d x^{-} \int d^{2} \mathbf{x}_{\perp} T^{++} \mathbf{x}_{\perp} .
$$

In terms of partonic transverse coordinates

$$
x_{i} \mathbf{r}_{\perp i}=x_{i} \mathbf{R}_{\perp}+\mathbf{b}_{\perp i},
$$

where the $\mathbf{r}_{\perp i}$ are the physical transverse position coordinates and $\mathbf{b}_{\perp i}$ frame independent internal coordinates, conjugate to the relative coordinates $\mathbf{k}_{\perp i}$. Thus, $\sum_{i=1}^{n} \mathbf{b}_{\perp i}=0$ and $\mathbf{R}_{\perp}=\sum_{i=1}^{n} x_{i} \mathbf{r}_{\perp i}$. The LFWF $\psi_{n}\left(x_{j}, \mathbf{k}_{\perp j}\right)$ can be expanded in terms of the $n-1$ independent coordinates $\mathbf{b}_{\perp j}, j=1,2, \ldots, n-1$

$$
\psi_{n}\left(x_{j}, \mathbf{k}_{\perp j}\right)=(4 \pi)^{\frac{(n-1)}{2}} \prod_{j=1}^{n-1} \int d^{2} \mathbf{b}_{\perp j} \exp \left(i \sum_{j=1}^{n-1} \mathbf{b}_{\perp j} \cdot \mathbf{k}_{\perp j}\right) \widetilde{\psi}_{n}\left(x_{j}, \mathbf{b}_{\perp j}\right) .
$$

The normalization is defined by

$$
\sum_{n} \prod_{j=1}^{n-1} \int d x_{j} d^{2} \mathbf{b}_{\perp j}\left|\widetilde{\psi}_{n}\left(x_{j}, \mathbf{b}_{\perp j}\right)\right|^{2}=1
$$

One of the important advantages of the light-front formalism is that current matrix elements can be represented without approximation as overlaps of light-front wavefunctions. In the case of the elastic space-like form factors, the matrix element of the $J^{+}$current only couples Fock states with the same number of constituents. If the charged parton $n$ is the active constituent struck by the current, and the quarks $i=1,2, \ldots, n-1$ are spectators, then the Drell-Yan West formula [42, 43, 44] in impact space is

$$
F\left(q^{2}\right)=\sum_{n} \prod_{j=1}^{n-1} \int d x_{j} d^{2} \mathbf{b}_{\perp j} \exp \left(i \mathbf{q}_{\perp} \cdot \sum_{j=1}^{n-1} x_{j} \mathbf{b}_{\perp j}\right)\left|\widetilde{\psi}_{n}\left(x_{j}, \mathbf{b}_{\perp j}\right)\right|^{2},
$$

corresponding to a change of transverse momenta $x_{j} \mathbf{q}_{\perp}$ for each of the $n-1$ spectators. This is a convenient form for comparison with AdS results, since the form factor is expressed in terms of the product of light-front wave functions with identical variables.

\section{Light-Front Mapping of String Amplitudes}

We can now establish an explicit connection between the AdS/CFT and the LF formulae. To make more transparent the holographic connection between $\mathrm{AdS}_{5}$ and 
the conformal quantum field theory defined at its asymptotic $z \rightarrow 0$ boundary, it is convenient to write the AdS metric (1) in terms of light front coordinates $x^{ \pm}=x^{0} \pm x^{3}$

$$
d s^{2}=\frac{R^{2}}{z^{2}}\left(d x^{+} d x^{-}-d \mathbf{x}_{\perp}^{2}-d z^{2}\right) .
$$

It is also useful to express (7) in terms of an effective single particle transverse distribution $\widetilde{\rho}$ [14]

$$
F\left(q^{2}\right)=2 \pi \int_{0}^{1} d x \frac{(1-x)}{x} \int \zeta d \zeta J_{0}\left(\zeta q \sqrt{\frac{1-x}{x}}\right) \tilde{\rho}(x, \zeta),
$$

where we have introduced the variable

$$
\zeta=\sqrt{\frac{x}{1-x}}\left|\sum_{j=1}^{n-1} x_{j} \mathbf{b}_{\perp j}\right|,
$$

representing the $x$-weighted transverse impact coordinate of the spectator system. On the other hand, the expression for the form factor in AdS space is represented as the overlap in the fifth dimension coordinate $z$ of the normalizable modes dual to the incoming and outgoing hadrons, $\Phi_{P}$ and $\Phi_{P^{\prime}}$, with the non-normalizable mode, $J(Q, z)=z Q K_{1}(z Q)$, dual to the external source [34]

$$
F\left(Q^{2}\right)=R^{3} \int \frac{d z}{z^{3}} \Phi_{P^{\prime}}(z) J(Q, z) \Phi_{P}(z) .
$$

If we compare (9) in impact space with the expression for the form factor in AdS space (11) for arbitrary values of $Q$ using the identity

$$
\int_{0}^{1} d x J_{0}\left(\zeta Q \sqrt{\frac{1-x}{x}}\right)=\zeta Q K_{1}(\zeta Q),
$$

then we can identify the spectator density function appearing in the light-front formalism with the corresponding AdS density

$$
\tilde{\rho}(x, \zeta)=\frac{R^{3}}{2 \pi} \frac{x}{1-x} \frac{|\Phi(\zeta)|^{2}}{\zeta^{4}} .
$$

Equation (13) gives a precise relation between string modes $\Phi(\zeta)$ in $\mathrm{AdS}_{5}$ and the QCD transverse charge density $\tilde{\rho}(x, \zeta)$. The variable $\zeta$ represents a measure of the transverse separation between point-like constituents, and it is also the holographic variable $z$ characterizing the string scale in AdS. Consequently the AdS string mode $\Phi(z)$ can be regarded as the probability amplitude to find $n$ partons at transverse 
impact separation $\zeta=z$. Furthermore, its eigenmodes determine the hadronic spectrum 14 .

In the case of a two-parton constituent bound state, the correspondence between the string amplitude $\Phi(z)$ and the light-front wave function $\widetilde{\psi}(x, \mathbf{b})$ is expressed in closed form 14

$$
|\widetilde{\psi}(x, \zeta)|^{2}=\frac{R^{3}}{2 \pi} x(1-x) \frac{|\Phi(\zeta)|^{2}}{\zeta^{4}},
$$

where $\zeta^{2}=x(1-x) \mathbf{b}_{\perp}^{2}$. Here $\mathbf{b}_{\perp}$ is the impact separation and Fourier conjugate to $\mathbf{k}_{\perp}$.

In presence of the dilaton background $\varphi=\kappa^{2} z^{2}$ the form factor in AdS space has the additional term $e^{-\kappa^{2} z^{2}}$ in the metric

$$
F\left(Q^{2}\right)=R^{3} \int \frac{d z}{z^{3}} e^{-\kappa^{2} z^{2}} \Phi(z) J_{\kappa}(Q, z) \Phi(z),
$$

to be properly normalized to the charge operator at $Q=0$. The non-normalizable electromagnetic mode $J_{\kappa}(Q, z)$ in 15

$$
J_{\kappa}(Q, z)=\Gamma\left(1+\frac{Q^{2}}{4 \kappa^{2}}\right) U\left(\frac{Q^{2}}{4 \kappa^{2}}, 0, \kappa^{2} z^{2}\right),
$$

couples to the dilaton field as it propagates in $\mathrm{AdS}$ space and $U(a, b, c)$ is the confluent hypergeometric function with the integral representation

$$
\Gamma(a) U(a, b, z)=\int_{0}^{\infty} e^{-z t} t^{a-1}(1+t)^{b-a-1} d t .
$$

In the large $Q^{2}$ limit, $Q^{2} \gg 4 \kappa^{2}$ we find that $J_{\kappa}(Q, z) \rightarrow z Q K_{1}(z Q)$. Thus, for large transverse momentum the current decouples from the dilaton background, and we recover our previous results for the ultraviolet behavior of matrix elements. When summed over all Fock states the Drell-Yan-West (DYW) formula gives an exact result. The formula describes the coupling of the free electromagnetic current to the elementary constituents in the interaction representation. In the presence of a dilaton field in AdS space, the electromagnetic AdS mode is no longer dual to a the free quark current, but dual to a dressed current, i.e., a hadronic electromagnetic current including virtual $\bar{q} q$ pairs and thus confined. We thus expect that the modified mapping corresponds to the presence of higher Fock states in the hadron.

\subsection{Holographic Light-Front Representation}

The mapping of $z$ from AdS space to $\zeta$ in the LF space allows the equations of motion in AdS space to be recast in the form of a light-front Hamiltonian equation [40]

$$
H_{L F}|\phi\rangle=\mathcal{M}^{2}|\phi\rangle
$$


a remarkable result which allows the discussion of the AdS/CFT solutions in terms of light-front equations in physical 3+1 space-time. By substituting $\phi(\zeta)=\left(\frac{\zeta}{R}\right)^{-3 / 2} \Phi(\zeta)$, in the AdS wave equation describing the propagation of scalar modes in AdS space

$$
\left[z^{2} \partial_{z}^{2}-(d-1) z \partial_{z}+z^{2} \mathcal{M}^{2}-(\mu R)^{2}\right] \Phi(z)=0
$$

we find an effective Schrödinger equation as a function of the weighted impact variable $\zeta$

$$
\left[-\frac{d^{2}}{d \zeta^{2}}+V(\zeta)\right] \phi(\zeta)=\mathcal{M}^{2} \phi(\zeta),
$$

with the effective potential $V(\zeta) \rightarrow-\left(1-4 L^{2}\right) / 4 \zeta^{2}$ in the conformal limit, where we identity $\zeta$ with the fifth dimension $z$ of AdS space: $\zeta=z$. We have written above $(\mu R)^{2}=-4+L^{2}$. The solution to 20 is $\phi(z)=z^{-\frac{3}{2}} \Phi(z)=C z^{\frac{1}{2}} J_{L}(z \mathcal{M})$. This equation reproduces the AdS/CFT solutions for mesons with relative orbital angular momentum $L$. The holographic hadronic light-front wave functions $\phi(\zeta)=\langle\zeta \mid \phi\rangle$ are normalized according to

$$
\langle\phi \mid \phi\rangle=\int d \zeta|\langle\zeta \mid \phi\rangle|^{2}=1
$$

and represent the probability amplitude to find $n$-partons at transverse impact separation $\zeta=z$. Its eigenmodes determine the hadronic mass spectrum.

The effective wave equation (20) is a relativistic light-front equation defined at $x^{+}=0$. The AdS metric $d s^{2}(8)$ is invariant if $\mathbf{x}_{\perp}^{2} \rightarrow \lambda^{2} \mathbf{x}_{\perp}^{2}$ and $z \rightarrow \lambda z$ at equal light-front time. The Casimir operator for the rotation group $S O(2)$ in the transverse light-front plane is $L^{2}$. This shows the natural holographic connection to the light front.

The lowest stable state $L=0$ is determined by the Breitenlohner-Freedman bound [45]. Its eigenvalues are set by the boundary conditions at $\phi\left(z=1 / \Lambda_{\mathrm{QCD}}\right)=0$ and are given in terms of the roots of Bessel functions: $\mathcal{M}_{L, k}=\beta_{L, k} \Lambda_{\mathrm{QCD}}$. Normalized LFWFs $\widetilde{\psi}_{L, k}$ follow from 14

$$
\widetilde{\psi}_{L, k}(x, \zeta)=B_{L, k} \sqrt{x(1-x)} J_{L}\left(\zeta \beta_{L, k} \Lambda_{\mathrm{QCD}}\right) \theta\left(z \leq \Lambda_{\mathrm{QCD}}^{-1}\right),
$$

where $B_{L, k}=\Lambda_{\mathrm{QCD}} / \sqrt{\pi} J_{1+L}\left(\beta_{L, k}\right)$. The resulting wavefunctions depicted in Fig. 1] display confinement at large interquark separation and conformal symmetry at short distances, reproducing dimensional counting rules for hard exclusive processes and the scaling and conformal properties of the LFWFs at high relative momenta in agreement with perturbative QCD.

Since they are complete and orthonormal, these AdS/CFT model wavefunctions can be used as an initial ansatz for a variational treatment or as a basis for the diagonalization of the light-front QCD Hamiltonian. We are now in fact investigating 

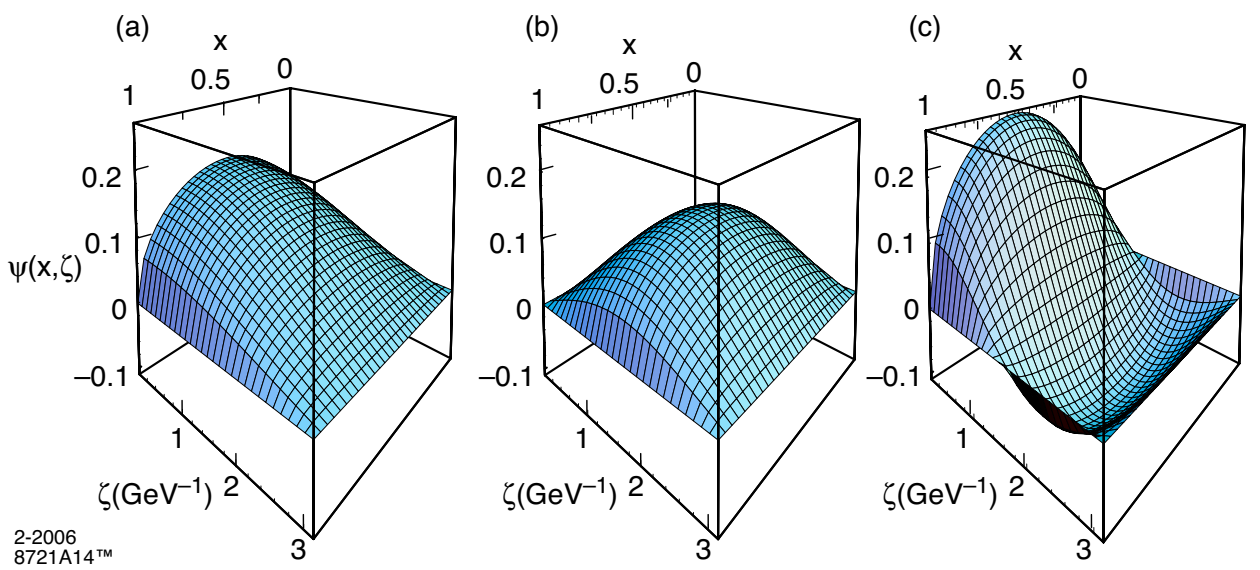

Figure 1: AdS/QCD Predictions for the light-front wavefunctions of a meson in the hard-wall model: (a) $n=0, L=0$, (b) $n=0, L=1$, (c) $n=1, L=0$.

this possibility with J. Vary and A. Harinandrath. Alternatively, one can introduce confinement by adding a two-dimensional harmonic oscillator potential $\kappa^{4} \zeta^{2}$ in the LF transverse plane to the conformal kernel in Eq. 20p. One can also introduce nonzero quark masses for the meson. The procedure is straightforward in the $\mathbf{k}_{\perp}$ representation by using the substitution $\frac{\mathbf{k}_{\perp}^{2}}{x(1-x)} \rightarrow \frac{\mathbf{k}_{\perp}^{2}+m_{1}^{2}}{x}+\frac{\mathbf{k}_{\perp}^{2}+m_{2}^{2}}{1-x}$.

\section{Integrability of AdS/CFT Equations}

The integrability methods of citeInfeld:1941 find a remarkable application in the AdS/CFT correspondence. Integrability follows if the equations describing a physical model can be factorized in terms of linear operators. These ladder operators generate all the eigenfunctions once the lowest mass eigenfunction is known. In holographic QCD, the conformally invariant $3+1$ light-front differential equations can be expressed in terms of ladder operators and their solutions can then be expressed in terms of analytical functions. In the conformal limit the ladder algebra for bosonic $(B)$ or fermionic $(F)$ modes is given in terms of the operator $\left(\Gamma^{B}=1, \quad \Gamma^{F}=\gamma_{5}\right)$

$$
\Pi_{\nu}^{B, F}(\zeta)=-i\left(\frac{d}{d \zeta}-\frac{\nu+\frac{1}{2}}{\zeta} \Gamma^{B, F}\right)
$$

and its adjoint

$$
\Pi_{\nu}^{B, F}(\zeta)^{\dagger}=-i\left(\frac{d}{d \zeta}+\frac{\nu+\frac{1}{2}}{\zeta} \Gamma^{B, F}\right),
$$

with commutation relations

$$
\left[\Pi_{\nu}^{B, F}(\zeta), \Pi_{\nu}^{B, F}(\zeta)^{\dagger}\right]=\frac{2 \nu+1}{\zeta^{2}} \Gamma^{B, F} .
$$


For $\nu \geq 0$ the Hamiltonian is written as a bilinear form $H_{L C}^{B, F}=\Pi_{\nu}^{B, F^{\dagger}} \Pi_{\nu}^{B, F}$. In the fermionic case the eigenmodes also satisfy a first order LF Dirac equation. For bosonic modes, the lowest stable state $\nu=0$ corresponds to the Breitenlohner-Freedman bound. Higher orbital states are constructed from the $L$-th application of the raising operator $a^{\dagger}=-i \Pi^{B}$ on the ground state.

\section{Hadronic Spectra in AdS/QCD}

The holographic model based on truncated AdS space can be used to obtain the hadronic spectrum of light quark $q \bar{q}, q q q$ and $g g$ bound states. Specific hadrons are identified by the correspondence of the amplitude in the fifth dimension with the twist dimension of the interpolating operator for the hadron's valence Fock state, including its orbital angular momentum excitations. Bosonic modes with conformal dimension $2+L$ are dual to the interpolating operator $\mathcal{O}_{\tau+L}$ with $\tau=2$. For fermionic modes $\tau=3$. For example, the set of three-quark baryons with spin $1 / 2$ and higher is described by the light-front Dirac equation

$$
\left(\alpha \Pi^{F}(\zeta)-\mathcal{M}\right) \psi(\zeta)=0
$$

where $i \alpha=\left(\begin{array}{cc}0 & I \\ -I & 0\end{array}\right)$ in the Weyl representation. The solution is

$$
\psi(\zeta)=C \sqrt{\zeta}\left[J_{L+1}(\zeta \mathcal{M}) u_{+}+J_{L+2}(z \mathcal{M}) u_{-}\right]
$$

with $\gamma_{5} u_{ \pm}=u_{ \pm}$. A discrete four-dimensional spectrum follows when we impose the boundary condition $\psi_{ \pm}\left(\zeta=1 / \Lambda_{\mathrm{QCD}}\right)=0: \mathcal{M}_{\alpha, k}^{+}=\beta_{\alpha, k} \Lambda_{\mathrm{QCD}}, \quad \mathcal{M}_{\alpha, k}^{-}=\beta_{\alpha+1, k} \Lambda_{\mathrm{QCD}}$ with a scale-independent mass ratio[28].

Figure 2(a) shows the predicted orbital spectrum of the nucleon states and Fig. 2 (b) the $\Delta$ orbital resonances. The spin-3/2 trajectories are determined from the corresponding Rarita-Schwinger equation. The solution of the spin-3/2 for polarization along Minkowski coordinates, $\psi_{\mu}$, is similar to the spin- $1 / 2$ solution. The data for the baryon spectra are from [47]. The internal parity of states is determined from the $\mathrm{SU}(6)$ spin-flavor symmetry. Since only one parameter, the QCD mass scale $\Lambda_{\mathrm{QCD}}$, is introduced, the agreement with the pattern of physical states is remarkable. In particular, the ratio of $\Delta$ to nucleon trajectories is determined by the ratio of zeros of Bessel functions. The predicted mass spectrum in the truncated space model is linear $M \propto L$ at high orbital angular momentum, in contrast to the quadratic dependence $M^{2} \propto L$ in the usual Regge parameterization. One can obtain $M^{2} \propto(L+n)$ dependence in the holographic model by the introduction of a harmonic potential $\kappa^{2} z^{2}$ in the AdS wave equations [38]. This result can also be obtained by extending the 


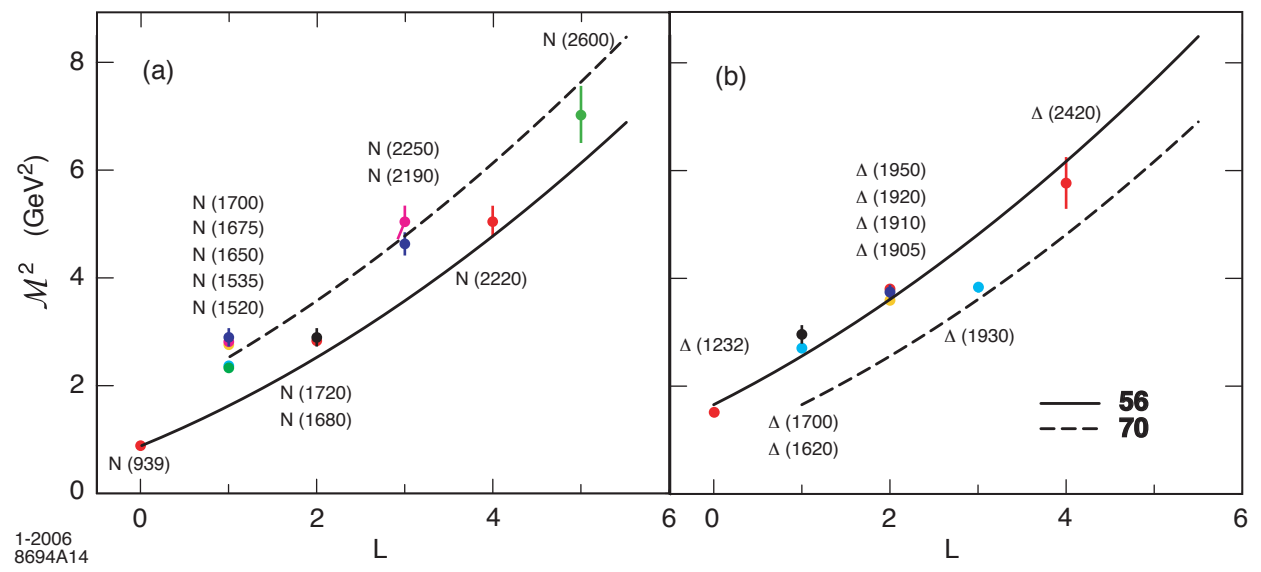

Figure 2: Predictions for the light baryon orbital spectrum for $\Lambda_{Q C D}=0.25 \mathrm{GeV}$. The $\mathbf{5 6}$ trajectory corresponds to $L$ even $P=+$ states, and the $\mathbf{7 0}$ to $L$ odd $P=-$ states.

conformal algebra written above. An account of the extended algebraic holographic model and a possible supersymmetric connection between the bosonic and fermionic operators used in the holographic construction will be described elsewhere.

\section{Analytic Results for Hadronic Observables in AdS/QCD}

\subsection{Hadronic Form Factors of Arbitrary Twist}

A string mode $\phi_{\tau}$ which couples to a local hadronic interpolating operator of twist $\tau$ defined at the asymptotic boundary of AdS space has scaling dimension $\tau, \Phi_{\tau} \sim z^{\tau}$, as $z \rightarrow 0$. In the soft-wall model a normalized string mode $\Phi_{\tau}$ is given by

$$
\Phi_{\tau}(z)=\frac{1}{R^{3 / 2}} \sqrt{\frac{2}{\Gamma(\tau-1)}} \kappa^{\tau-1} z^{\tau} .
$$

for $n=0$. It is convenient to perform the change of variable $t=\frac{x}{1-x}$ in the integral representation for the bulk-to-boundary propagator (16). Upon integration by parts the resulting expression, there follows

$$
J_{\kappa}(Q, z)=\kappa^{2} z^{2} \int_{0}^{1} \frac{d x}{(1-x)^{2}} x^{\frac{Q^{2}}{4 \kappa^{2}}} e^{-\kappa^{2} z^{2} x /(1-x)}
$$

the result found by Grigoryan and Radyuskin in Ref. [17]. We can then find an analytical expression for the form factor of a hadronic state of arbitrary twist $\tau$ by 
substituting the integral representation $(29)$ for $J_{\kappa}(q, z)$ in $(15)$. We find the result

$$
F\left(Q^{2}\right)=\Gamma(\tau) \frac{\Gamma\left(1+\frac{Q^{2}}{4 \kappa^{2}}\right)}{\Gamma\left(\tau+\frac{Q^{2}}{4 \kappa^{2}}\right)} .
$$

In the absence of anomalous dimensions, the twist is an integer, $\tau=N$, and we can simplify 30 by using the recurrence formula

$$
\Gamma(N+z)=(N-1+z)(N-2+z) \ldots(1+z) \Gamma(1+z) .
$$

We find

$$
\begin{aligned}
& F\left(Q^{2}\right)=\frac{1}{1+\frac{Q^{2}}{4 \kappa^{2}}}, \quad N=2, \\
& F\left(Q^{2}\right)=\frac{2}{\left(1+\frac{Q^{2}}{4 \kappa^{2}}\right)\left(2+\frac{Q^{2}}{4 \kappa^{2}}\right)}, \quad N=3, \\
& F\left(Q^{2}\right)=\frac{(N-1) !}{\left(1+\frac{Q^{2}}{4 \kappa^{2}}\right)\left(2+\frac{Q^{2}}{4 \kappa^{2}}\right) \cdots\left(N-1+\frac{Q^{2}}{4 \kappa^{2}}\right)}, N
\end{aligned}
$$

which is expressed as a product of $N-1$ poles, corresponding to the first $N-1$ states along the vector meson radial trajectory. For large $Q^{2}$ it follows that

$$
F\left(Q^{2}\right) \rightarrow(N-1) !\left[\frac{4 \kappa^{2}}{Q^{2}}\right]^{(N-1)},
$$

and we recover the conformal power-law counting rules for hard scattering [48, 49, 50].

\subsection{Meson Form Factors}

As we have discussed, hadron form factors can be predicted in AdS from the overlap of the normalizable modes dual to the incoming and outgoing hadrons with the bulk-toboundary propagator, $J(Q, z)$, dual to the external source, or equivalently by using the DYW formula in physical space-time. For example, we can compute the pion form factor from the AdS expressions (11) and (15) for the hadronic string modes $\Phi_{\pi}$ in the hard-wall (HW)

$$
\Phi_{\pi}^{H W}(z)=\frac{\sqrt{2} \Lambda_{Q C D}}{R^{3 / 2} J_{1}\left(\beta_{0,1}\right)} z^{2} J_{0}\left(z \beta_{0,1} \Lambda_{Q C D}\right),
$$

and soft-wall (SW) model

$$
\Phi_{\pi}^{S W}(z)=\frac{\sqrt{2} \kappa}{R^{3 / 2}} z^{2}
$$


respectively. Since the pion mode couples to a twist-two boundary interpolating operator which creates a two-component hadronic bound state, the form factor is given in the SW model by the simple monopole form (32) corresponding to $n=2$

$$
F_{\pi}\left(Q^{2}\right)=\frac{4 \kappa^{2}}{4 \kappa^{2}+Q^{2}}
$$

The hadronic scale is evaluated by fitting the space-like data for the form factor as shown in Figure 3, where we plot the product $Q^{2} F_{\pi}\left(Q^{2}\right)$ for the soft and hard-wall holographic models. Both models would seem to describe the overall behavior of the space-like data; however, when the low energy data is examined in detail, the SW model gives a noticeable better description as shown in Figure 4. When the

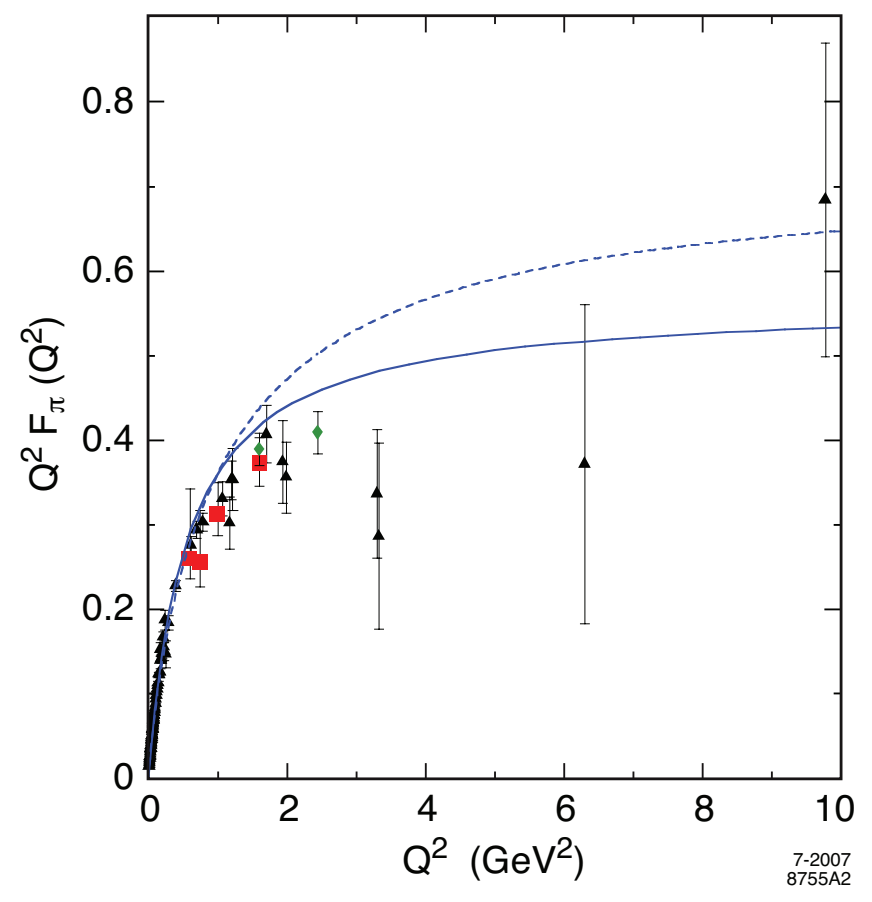

Figure 3: Space-like scaling behavior for $Q^{2} F_{\pi}\left(Q^{2}\right)$ as a function of $Q^{2}=-q^{2}$. The continuous line is the prediction of the soft-wall model for $\kappa=0.375 \mathrm{GeV}$. The dashed line is the prediction of the hard-model for $\Lambda_{Q C D}=0.22 \mathrm{GeV}$. The black triangles is the data compilation from Baldini et al. [51], the red boxes are JLAB 1 data [52] and cobalt green diamonds are JLAB 2 data [53].

results for the pion form factor are analytically continued to the time-like region, $q^{2} \rightarrow-q^{2}$ we obtain the results shown in Figure 5 for $\log \left(\left|F_{\pi}\left(q^{2}\right)\right|\right)$. The monopole form of the SW model exhibits a pole at the $\rho$ mass and reproduces well the $\rho$ peak with $M_{\rho}=4 \kappa^{2}=750 \mathrm{MeV}$. In the strongly coupled semiclassical gauge/gravity limit hadrons have zero widths and are stable. The form factor accounts for the scaling 


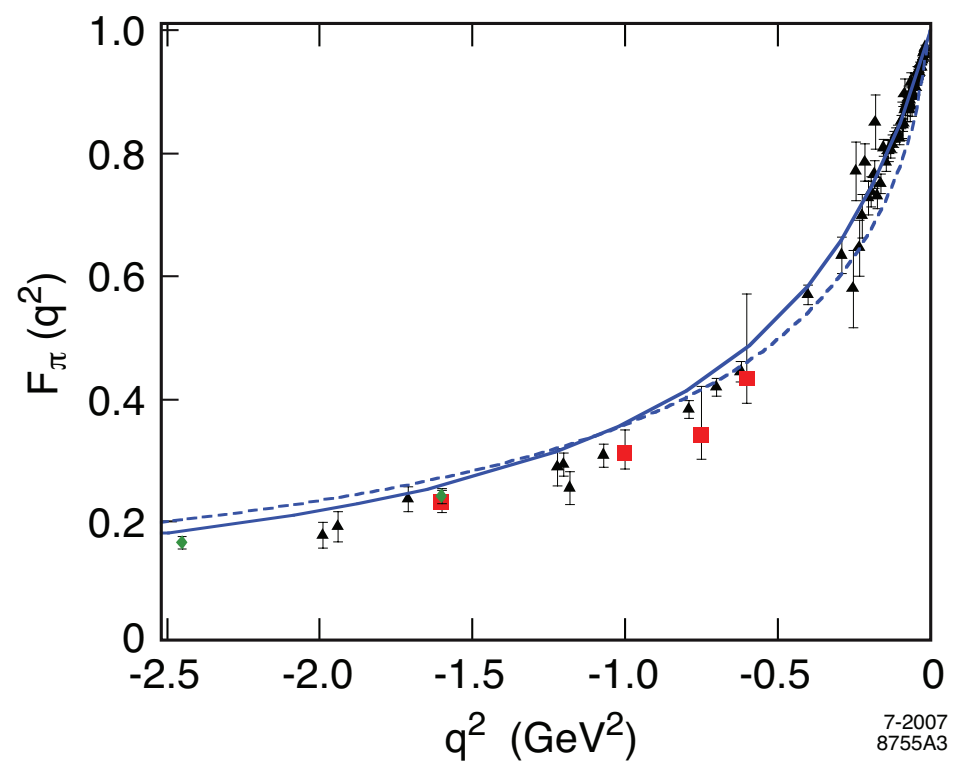

Figure 4: Space-like behavior of the pion form factor $F_{\pi}\left(q^{2}\right)$ as a function of $q^{2}$ for $\kappa=0.375 \mathrm{GeV}$ and $\Lambda_{Q C D}=0.22 \mathrm{GeV}$. Continuous line: soft-wall model, dashed line: hard-wall model. Black (triangle) is the data compilation from Baldini et al. [51], red (box) is JLAB 1 [52] and cobalt green diamonds are JLAB 2 [53].

behavior in the space-like region, but does not give rise to the additional structure found in the time-like region, since the $\rho$ pole saturates $100 \%$ the monopole form $(38)$.

\subsection{Contributions to Meson Form Factors and Structure Func- tions at Large Momentum Transfer in AdS/QCD}

The form factor of a hadron at large $Q^{2}$ arises from the small $z$ kinematic domain in AdS space. According to the AdS/CFT duality, this corresponds to small distances $x^{\mu} x_{\mu} \sim 1 / Q^{2}$ in physical space-time, the domain where the current matrix elements are controlled by the conformal twist-dimension, $\Delta$, of the hadron's interpolating operator. In the case of the front form, where $x^{+}=0$, this corresponds to small transverse separation $x^{\mu} x_{\mu}=-\mathbf{x}_{\perp}^{2}$.

As we have shown [14], one can use holography to map the functional from of the string modes $\Phi(z)$ in AdS space to the light front wavefunctions in physical space time by identifying $z$ with the transverse variable $\zeta=\sqrt{\frac{x}{1-x}}\left|\vec{\eta}_{\perp}\right|$. Here $\vec{\eta}_{\perp}=\sum_{i=1}^{n-1} x_{i} \mathbf{b}_{\perp i}$ is the weighted impact separation, summed over the impact separation of the spectator constituents. The leading large- $Q^{2}$ behavior of form factors in AdS/QCD arises from small $\zeta \sim 1 / Q$, corresponding to small transverse separation. 


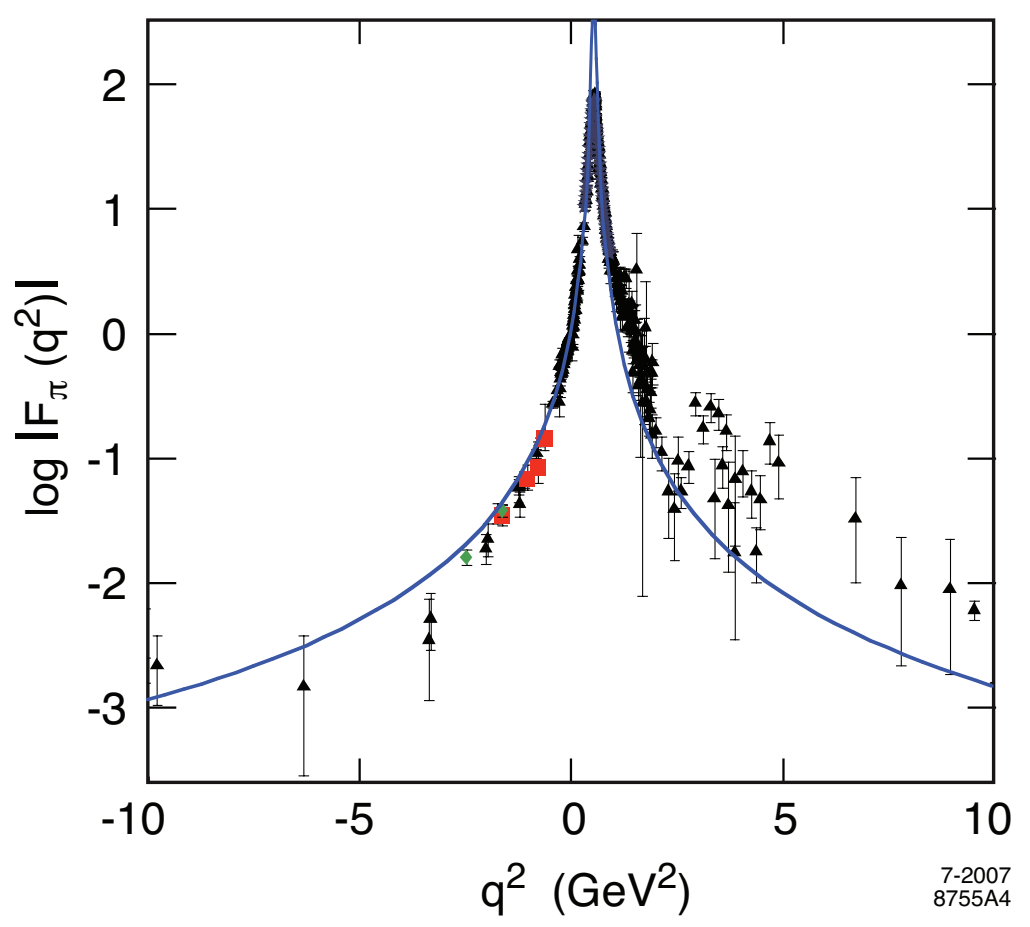

Figure 5: Space and time-like behavior of the pion form factor $\log \left(\left|F_{\pi}\left(q^{2}\right)\right|\right)$ as a function of $q^{2}$ for $\kappa=0.375 \mathrm{GeV}$ in the soft-wall model. Black (triangle) is the data compilation from Baldini et al. [51], red (box) is JLAB 1 [52] and cobalt green diamonds are JLAB 2 [53].

For the case of a meson with two constituents the form factor can be written in terms of an effective light-front transverse density in impact space

$$
F\left(q^{2}\right)=\int_{0}^{1} d x \int d b^{2} \widetilde{\rho}(x, b, Q),
$$

with $\widetilde{\rho}(x, b, Q)=\pi J_{0}(b Q(1-x))|\widetilde{\psi}(x, b)|^{2}$ and $b=\left|\mathbf{b}_{\perp}\right|$. The kinematics are illustrated in figure 6 for the case of a meson with two constituents in the soft-wall model

$$
\widetilde{\psi}_{q \bar{q} / \pi}\left(x, \mathbf{b}_{\perp}\right)=\frac{\kappa}{\sqrt{\pi}} \sqrt{x(1-x)} e^{-\frac{1}{2} \kappa^{2} x(1-x) \mathbf{b}_{\perp}^{2}},
$$

where the Gaussian form of the LFWF at large $Q^{2}$ favors short-distance configurations with small $\zeta^{2}=b_{\perp}^{2} x(1-x) \sim 1 / Q^{2}$. Since we are mainly interested in studying the contribution from different regions to the form factor at large $Q^{2}$, we have replaced the modified bulk-to-boundary propagator $J_{\kappa}(Q, z)$ (16) by its large $Q^{2}$ form $z Q K_{1}(z Q)$. One sees a shift of the integrand $\tilde{\rho}(x, b, Q)$ toward small $\left|\mathbf{b}_{\perp}\right|$ and small $1-x$ at high $Q^{2}$. A similar behavior is observed for the LFWF obtained from the hard wall model. 

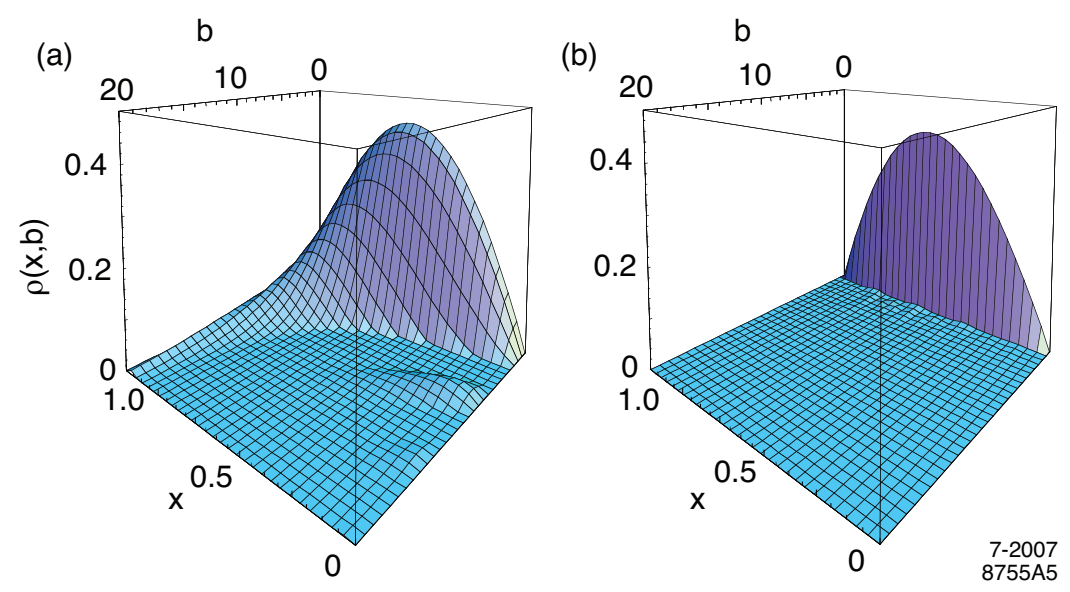

Figure 6: Effective partonic density $\rho(x, b, Q)$ in terms of the longitudinal momentum fraction $x$, the transverse relative impact variable $b=\left|\mathbf{b}_{\perp}\right|$ and momentum transfer $Q$ for the soft wall model. As $Q$ increases the distribution becomes increasingly important near $x=1$ and $\mathbf{b}_{\perp}=0$. This is illustrated in (a) for $Q=1 \mathrm{GeV} / \mathrm{c}$. At very large $Q$ (figure (b)), the distribution is peaked towards $\mathbf{b}_{\perp}=0$. The value of $\kappa$ is $0.375 \mathrm{GeV}$.

\subsection{The Meson Structure Function}

The pion structure function $q_{\pi}\left(x, Q^{2}\right)$ is computed by integrating the square of the pion light-front wave function up to the scale $Q^{2}$

$$
q_{\pi}\left(x, Q^{2}\right)=\int^{Q^{2}} \frac{d^{2} \mathbf{k}_{\perp}}{16 \pi^{3}}\left|\psi_{\bar{q} q / \pi}\left(x, \mathbf{k}_{\perp}\right)\right|^{2}
$$

where the LFWF in $\mathbf{k}_{\perp}$ space

$$
\psi_{\bar{q} q / \pi}\left(x, \mathbf{k}_{\perp}\right)=\frac{4 \pi}{\kappa \sqrt{x(1-x)}} e^{-\frac{\mathbf{k}_{\perp}^{2}}{2 \kappa^{2} x(1-x)}} .
$$

is the Fourier transform (5) for a two-parton bound state. We find

$$
q_{\pi}\left(x, Q^{2}\right)=1-e^{-\frac{Q^{2}}{\kappa^{2} x(1-x)}},
$$

In the large $Q^{2}$ limit $q_{\pi}\left(x, Q^{2} \rightarrow \infty\right) \equiv q_{\pi}(x)=1$, which is the behavior of a strongly coupled theory found in $\mathrm{QCD}(1+1)$ [54]. Identical results [55] are obtained for the pion in the hard wall model.

\subsection{The Pion Decay Constant}

The pion decay constant is given by the matrix element of the axial isospin current $J^{\mu 5 a}$ between a physical pion and the vacuum state [56] $\left\langle 0\left|J_{W}^{+}(0)\right| \pi^{-}\left(P^{+}, \vec{P}_{\perp}\right)\right\rangle$, where 
$J_{W}^{+}$is the flavor changing weak current. Only the valence state with $L_{z}=0, S_{z}=0$, contributes to the decay of the $\pi^{ \pm}$. Expanding the hadronic initial state in the decay amplitude into its Fock components we find

$$
f_{\pi}=2 \sqrt{N_{C}} \int_{0}^{1} d x \int \frac{d^{2} \vec{k}_{\perp}}{16 \pi^{3}} \psi_{\bar{q} q / \pi}\left(x, k_{\perp}\right) .
$$

This light-cone equation allows the exact computation of the pion decay constant in terms of the valence pion light-front wave function [6].

\subsection{The Meson Distribution Amplitude}

The meson distribution amplitude $\phi(x, Q)$ is defined as [4]

$$
\phi(x, Q)=\int^{Q^{2}} \frac{d^{2} \mathbf{k}_{\perp}}{16 \pi^{3}} \psi\left(x, \mathbf{k}_{\perp}\right) .
$$

It follows that

$$
\phi_{\pi}(x, Q \rightarrow \infty)=\frac{4}{\sqrt{3} \pi} f_{\pi} \sqrt{x(1-x)}
$$

with

$$
f_{\pi}=\frac{1}{8} \sqrt{\frac{3}{2}} R^{3 / 2} \lim _{\zeta \rightarrow 0} \frac{\Phi(\zeta)}{\zeta^{2}},
$$

since $\phi(x, Q \rightarrow \infty) \rightarrow \widetilde{\psi}\left(x, \mathbf{b}_{\perp} \rightarrow 0\right) / \sqrt{4 \pi}$ and $\Phi_{\pi} \sim \zeta^{2}$ as $\zeta \rightarrow 0$. The pion decay constant depends only on the behavior of the AdS string mode near the asymptotic boundary, $\zeta=z=0$ and the mode normalization. For the truncated-space (TS) pion mode we find $f_{\pi}^{T S}=\frac{\sqrt{3}}{8 J_{1}\left(\beta_{0, k}\right)} \Lambda_{\mathrm{QCD}}=83.4 \mathrm{MeV}$, for $\Lambda_{Q C D}=0.22 \mathrm{MeV}$. The corresponding result for the transverse harmonic oscillator (HO) pion mode is $f_{\pi}^{H O}=$ $\frac{\sqrt{3}}{8} \kappa=86.6 \mathrm{MeV}$, for $\kappa=0.375 \mathrm{GeV}$. The values of $\Lambda_{Q C D}$ and $\kappa$ are determined from the space-like form factor data as discussed above. The experimental result for $f_{\pi}$ is extracted from the rate of weak $\pi$ decay and has the value $f_{\pi}=92.4 \mathrm{MeV}$ [47].

It is interesting to note that the pion distribution amplitude predicted by AdS/QCD 46) has a quite different $x$-behavior than the asymptotic distribution amplitude predicted from the PQCD evolution [4] of the pion distribution amplitude $\phi_{\pi}(x, Q \rightarrow$ $\infty)=\sqrt{3} f_{\pi} x(1-x)$. The broader shape of the pion distribution increases the magnitude of the leading twist perturbative QCD prediction for the pion form factor by a factor of 16/9 compared to the prediction based on the asymptotic form, bringing the NLO PQCD prediction close to the empirical pion form factor [57]. The Fermilab E791 measurements [58] of the $x$ and $k_{\perp}$ fall-off of the diffractive dijet cross section $\pi A \rightarrow$ Jet Jet $A$ also suggest a broad pion distribution amplitude for jet transverse momenta $k_{\perp}^{2}<2 \mathrm{GeV}^{2}$. 


\subsection{The Nucleon Dirac Form Factors}

As a final example we compute the spin non-flip nucleon form factor in the soft wall model. Consider the spin non-flip form factors

$$
\begin{aligned}
& F_{+}\left(Q^{2}\right)=g_{+} \int \frac{d z}{z} e^{-\kappa^{2} z^{2}} J_{\kappa}(Q, z)\left|\Psi_{+}(z)\right|^{2}, \\
& F_{-}\left(Q^{2}\right)=g_{-} \int \frac{d z}{z} e^{-\kappa^{2} z^{2}} J_{\kappa}(Q, z)\left|\Psi_{-}(z)\right|^{2},
\end{aligned}
$$

where the effective charges $g_{+}$and $g_{-}$are determined from the spin-flavor structure of the theory. We choose the struck quark to have $S^{z}=+1 / 2$. The two AdS solutions $\Psi_{+}$and $\Psi_{-}$correspond to nucleons with total angular momentum $J^{z}=+1 / 2$ and $-1 / 2$. For the $S U(6)$ spin-flavor symmetry

$$
\begin{aligned}
F_{1}^{p}\left(Q^{2}\right) & =\int \frac{d z}{z} e^{-\kappa^{2} z^{2}} J_{\kappa}(Q, z)\left|\Psi_{+}(\zeta)\right|^{2} \\
F_{1}^{n}\left(Q^{2}\right) & =-\frac{1}{3} \int \frac{d z}{z} e^{-\kappa^{2} z^{2}} J_{\kappa}(Q, z)\left[\left|\Psi_{+}(z)\right|^{2}-\left|\Psi_{-}(z)\right|^{2}\right],
\end{aligned}
$$

where $F_{1}^{p}(0)=1, \quad F_{1}^{n}(0)=0$. The bulk-to-boundary propagator $J_{\kappa}(Q, z)$ is the solution (16) of the AdS wave equation for the external electromagnetic current, and the plus and minus components of the twist 3 nucleon mode in the soft wall model are

$$
\begin{gathered}
\Psi_{+}(z)=\frac{\sqrt{2} \kappa^{2}}{R^{3 / 2}} z^{3}, \\
\Psi_{-}(z)=\frac{\kappa^{3}}{R^{3 / 2}} z^{4} .
\end{gathered}
$$

The results for $Q^{4} F_{1}^{p}\left(Q^{2}\right)$ and $Q^{4} F_{1}^{n}\left(Q^{2}\right)$ follow from the analytic form for the form factors for any $\tau$ given in Section 7.1 and are shown in Figure 7 .

\section{Overview of Hard Exclusive Processes}

The leading power fall-off of the hard scattering amplitude follows from the conformal scaling of the underlying hard-scattering amplitude: $T_{H} \sim 1 / Q^{n-4}$, where $n$ is the total number of fields (quarks, leptons, or gauge fields) participating in the hard scattering [48, 49]. Thus the reaction is dominated by subprocesses and Fock states involving the minimum number of interacting fields. In the case of $2 \rightarrow 2$ scattering processes, this implies $d \sigma / d t(A B \rightarrow C D)=F_{A B \rightarrow C D}(t / s) / s^{n-2}$, where $n=N_{A}+N_{B}+$ $N_{C}+N_{D}$ and $n_{H}$ is the minimum number of constituents of $H$. These dimensional 

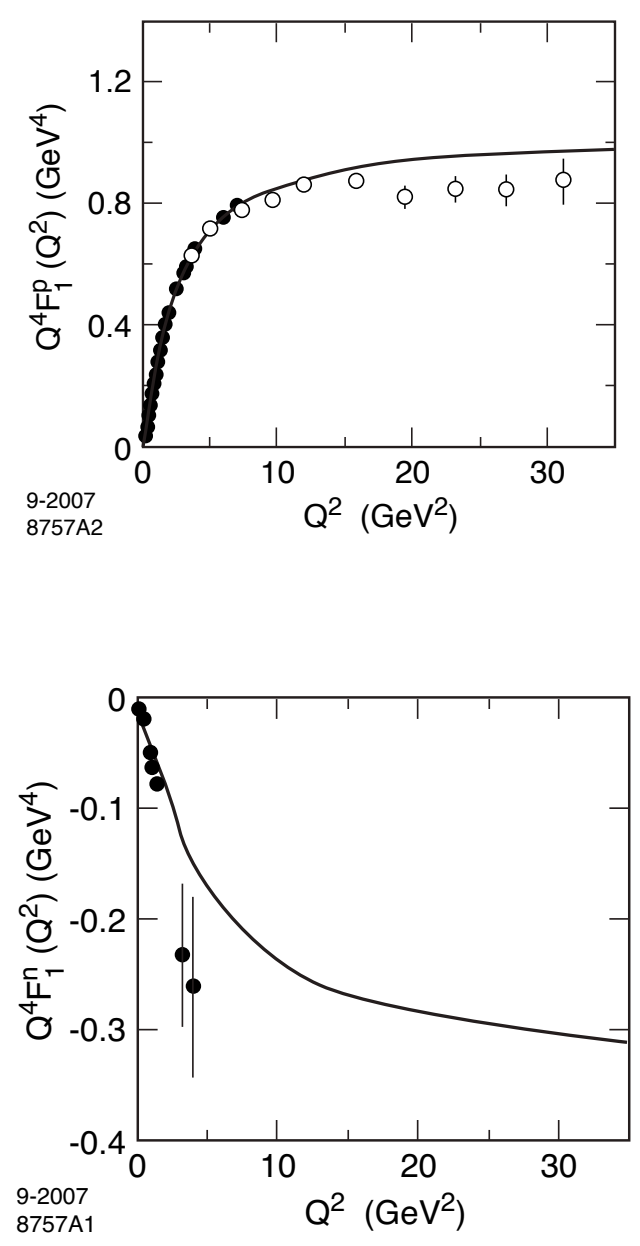

Figure 7: Predictions for $Q^{4} F_{1}^{p}\left(Q^{2}\right)$ and $Q^{4} F_{1}^{n}\left(Q^{2}\right)$ in the soft wall model for $\kappa=0.424$ $\mathrm{GeV}$. The data compilation is from Diehl [59].

counting rules are also a key feature of AdS/CFT [24]. The near-constancy of the effective QCD coupling at small scales helps explain the general empirical success of the dimensional counting rules for the near-conformal power law fall-off of form factors and fixed-angle scaling [60]. For example, measurements of pion photoproduction are consistent with dimensional counting $s^{7} d \sigma / d t\left(\gamma p \rightarrow \pi^{+} n\right) \sim$ constant at fixed CM angle for $s>7 \mathrm{GeV}$. The angular distributions seen in hard large CM angle scattering reactions are consistent with quark interchange, a result predicted by the hard wall AdS/QCD model. Reviews are given in refs. [61] and [62]. One sees the onset of perturbative QCD scaling behavior even for exclusive nuclear amplitudes such as deuteron photodisintegration (Here $n=1+6+3+3=13) s^{11} d \sigma / d t(\gamma d \rightarrow p n) \sim$ 
constant at fixed CM angle [63, 64, 65]. The measured deuteron form factor [66] also appears to follow the leading-twist QCD predictions 67] at large momentum transfers in the few $\mathrm{GeV}$ region. A measurement of $d \sigma / d t\left(\gamma d \rightarrow \Delta^{++} \Delta^{\prime}\right)$ in the scaling region can establish the role of "hidden-color" degrees of freedom 68] of the nuclear wavefunction in hard deuteron reactions.

Recently the Hall A collaboration [69] at JLab has reported a significant exception to the general empirical success of dimensional counting in fixed CM angle Compton scattering $d \sigma / d t(\gamma p \rightarrow \gamma p) \sim F\left(\theta_{C M}\right) / s^{8}$ instead of the predicted $1 / s^{6}$ scaling. The deviations from fixed-angle conformal scaling may be due to corrections from resonance contributions in the JLab energy range. It is interesting that the hadron form factor $R_{V}(t)$ [70], which multiplies the $\gamma q \rightarrow \gamma q$ amplitude is found by Hall-A to scale as $1 / t^{2}$, in agreement with the PQCD and AdS/CFT prediction. In addition the Belle measurement [71] of the timelike two-photon cross section $d \sigma / d t(\gamma \gamma \rightarrow p \bar{p})$ is consistent with $1 / s^{6}$ scaling.

Although large-angle proton-proton elastic scattering is well described by dimensional scaling $s^{10} d \sigma / d t(p p \rightarrow p p) \sim$ constant at fixed CM angle, extraordinarily large spin-spin correlations are observed [72]. The ratio of scattering cross sections for spinparallel and normal to the scattering plane versus spin-antiparallel reaches $R_{N N} \simeq 4$ in large angle $p p \rightarrow p p$ at $\sqrt{s} \simeq 5 \mathrm{GeV}$; this is a remarkable example of "exclusive transversity". Color transparency is observed at lower energies but it fails [73] at the same energy where $R_{N N}$ becomes large. In fact, these anomalies have a natural explanation [74] as a resonance effect related to the charm threshold in $p p$ scattering. Alternative explanations of the large spin correlation are discussed and reviewed in ref. [75]. Resonance formation is a natural phenomenon when all constituents are relatively at rest. For example, a resonance effect can occur due to the intermediate state $u$ uduudc $\bar{c}$ at the charm threshold $\sqrt{s}=5 \mathrm{GeV}$ in $p p$ collisions. Since the $c$ and $\bar{c}$ have opposite intrinsic parity, the resonance appears in the $L=J=S=1$ partial wave for $p p \rightarrow p p$ which is only allowed for spin-parallel and normal scattering $A_{N N}=1$ [74]. Resonance formation at the charm threshold also explains the dramatic quenching of color transparency seen in quasielastic pn scattering by the EVA BNL experiment 73] in the same kinematic region. The reason why these effects are so dramatic in $p p \rightarrow p p$ scattering is that the amplitude for the formation of an $u$ uduudc $\bar{c} s$-channel resonance in the intermediate state is of the same magnitude as the fast-falling background $p p \rightarrow p p$ PQCD amplitude from quark interchange at large CM angles: $M(p p \rightarrow p p) \sim 1 / u^{2} t^{2}$. We have predicted that the open charm cross section in $p p$ scattering will be of order of $1 \mu b$ at threshold [74].

Color transparency [76, 77] is a key property of color gauge theory, and it thus stands at the foundations of QCD. Color transparency has been confirmed in diffrac- 
tive dijet production [78], pion photoproduction [79] and vector meson electroproduction [80], but it is very important to also systematically validate it in large angle hadron scattering processes. One also expects similar novel QCD phenomena in largeangle photoproduction $\gamma p \rightarrow \pi N$ near the charm threshold, including the breakdown of color transparency and strong spin-spin correlations. These effects can be tested by measurements at the new JLab $12 \mathrm{GeV}$ facility, which would confirm resonance formation in a low partial wave in $\gamma p \rightarrow \pi N$ at $\sqrt{s} \simeq 4 \mathrm{GeV}$ due to attractive forces

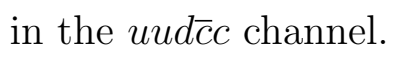

\section{Conclusions}

We have shown how the AdS/CFT correspondence between Anti-de Sitter space and conformal gauge provides an analytically tractable approximation to QCD in the regime where the QCD coupling is large and constant. In particular, there is an exact correspondence between the fifth-dimension coordinate $z$ of AdS space and an impact variable $\zeta$ which measures the invariant separation of the quark constituents within the hadron in ordinary space-time. This connection allows one to compute the analytic form of the frame-independent light-front wavefunctions of mesons and baryons, the fundamental entities which encode hadron properties and allow the computation of exclusive scattering amplitudes.

The phenomenology of the AdS/QCD model is just beginning, but it can be anticipated that it will have many applications to hadron dynamics. For example, the model LFWFs obtained from AdS/QCD provide a basis for understanding hadron structure functions and fragmentation functions at the amplitude level; the same wavefunctions also describe hadron formation from the coalescence of co-moving quarks. The spin correlations which underly single and double spin correlations are also described by the AdS/QCD eigensolutions. The AdS/QCD hadronic wavefunctions provide

predictions for the generalized parton distributions of hadrons and their weak decay amplitudes from first principles. The amplitudes relevant to diffractive reactions could also be computed. We also anticipate that the extension of the AdS/QCD formalism to heavy quarks will allow a great variety of heavy hadron phenomena to be analyzed from first principles.

\section{Acknowledgments}

This research was supported by the Department of Energy contract DE-AC0276SF00515. The research of GdT is supported in part by an Aportes grant from Florida Ice \& Farm. Invited talk, presented at the Workshop on Exclusive Reactions at High Momentum Transfer 21-24 May 2007, Newport News, Virginia. 


\section{References}

[1] P. A. M. Dirac, "Forms Of Relativistic Dynamics," Rev. Mod. Phys. 21, 392 (1949).

[2] S. J. Brodsky and D. S. Hwang, "Exact light-cone wavefunction representation of matrix elements of electroweak currents," Nucl. Phys. B 543, 239 (1999) arXiv:hep-ph/9806358.

[3] S. J. Brodsky, M. Diehl and D. S. Hwang, "Light-cone wavefunction representation of deeply virtual Compton scattering," Nucl. Phys. B 596, 99 (2001) arXiv:hep$\mathrm{ph} / 0009254$.

[4] G. P. Lepage and S. J. Brodsky, "Exclusive Processes In Quantum Chromodynamics: Evolution Equations For Hadronic Wave Functions And The Form-Factors Of Mesons," Phys. Lett. B 87, 359 (1979).

[5] A. V. Efremov and A. V. Radyushkin, "Factorization And Asymptotical Behavior Of Pion Form-Factor In QCD," Phys. Lett. B 94, 245 (1980).

[6] G. P. Lepage and S. J. Brodsky, "Exclusive Processes In Perturbative Quantum Chromodynamics," Phys. Rev. D 22, 2157 (1980).

[7] S. J. Brodsky and G. P. Lepage, "Exclusive Processes And The Exclusive Inclusive Connection In Quantum Chromodynamics," SLAC-PUB-2294 (1979).

[8] J. M. Maldacena, "The large N limit of superconformal field theories and supergravity," Adv. Theor. Math. Phys. 2, 231 (1998) [Int. J. Theor. Phys. 38, 1113 (1999)] [arXiv:hep-th/9711200]; S. S. Gubser, I. R. Klebanov and A. M. Polyakov, "Gauge theory correlators from non-critical string theory," Phys. Lett. B 428, 105 (1998) [arXiv:hep-th/9802109]; E. Witten, "Anti-de Sitter space and holography," Adv. Theor. Math. Phys. 2, 253 (1998) arXiv:hep-th/9802150|.

[9] L. von Smekal, R. Alkofer and A. Hauck, "The infrared behavior of gluon and ghost propagators in Landau gauge QCD," Phys. Rev. Lett. 79, 3591 (1997) arXiv:hep-ph/9705242.

[10] S. Furui and H. Nakajima, "Infrared features of unquenched finite temperature lattice Landau gauge QCD," arXiv:hep-lat/0612009; S. Furui and H. Nakajima, "Roles of the quark field in the infrared lattice Coulomb gauge and Landau gauge QCD," arXiv:0708.1421 [hep-lat]. 
[11] S. J. Brodsky, S. Menke, C. Merino and J. Rathsman, "On the behavior of the effective QCD coupling alpha(tau)(s) at low scales," Phys. Rev. D 67, 055008 (2003) arXiv:hep-ph/0212078.

[12] A. Deur, V. Burkert, J. P. Chen and W. Korsch, "Experimental determination of the effective strong coupling constant," Phys. Lett. B 650, 244 (2007) arXiv:hepph/0509113.

[13] J. M. Cornwall, "Dynamical Mass Generation In Continuum QCD," Phys. Rev. D 26, 1453 (1982).

[14] S. J. Brodsky and G. F. de Teramond, "Hadronic spectra and light-front wavefunctions in holographic QCD," Phys. Rev. Lett. 96, 201601 (2006) arXiv:hepph/0602252].

[15] G. F. de Teramond, "Mapping string states into partons: Form factors and the hadron spectrum in arXiv:hep-ph/0606143.

[16] H. R. Grigoryan and A. V. Radyushkin, "Form Factors and Wave Functions of Vector Mesons in Holographic QCD," Phys. Lett. B 650, 421 (2007) arXiv:hepph/0703069]; "Pion Form Factor in Chiral Limit of Hard-Wall AdS/QCD Model," arXiv:0709.0500 [hep-ph]; H. R. Grigoryan, "Dimension Six Correction to the Vector Sector of AdS/QCD Model," arXiv:0709.0939 [hep-ph].

[17] H. R. Grigoryan and A. V. Radyushkin, "Structure of Vector Mesons in Holographic Model with Linear Confinement," arXiv:0706.1543 [hep-ph].

[18] S. J. Brodsky and G. F. de Teramond, "AdS/CFT and QCD," arXiv:hepth/0702205.

[19] S. J. Brodsky and G. F. de Teramond, "Light-Front Dynamics and AdS/QCD: The Pion Form Factor in the Space- and Time-Like Regions," arXiv:0707.3859 [hep-ph].

[20] T. Huang and F. Zuo, "Couplings of the Rho Meson in a Holographic dual of QCD with Regge Trajectories," arXiv:0708.0936 [hep-ph].

[21] H. J. Kwee and R. F. Lebed, "Pion Form Factors in Holographic QCD," arXiv:0708.4054 [hep-ph].

[22] J. Polchinski and L. Susskind, "String theory and the size of hadrons," arXiv:hepth/0112204. 
[23] S. Hong, S. Yoon and M. J. Strassler, "On the couplings of vector mesons in AdS/QCD," JHEP 0604, 003 (2006) arXiv:hep-th/0409118.

[24] J. Polchinski and M. J. Strassler, "Hard scattering and gauge/string duality," Phys. Rev. Lett. 88, 031601 (2002) arXiv:hep-th/0109174].

[25] S. J. Brodsky and G. F. de Teramond, "Light-front hadron dynamics and AdS/CFT correspondence," Phys. Lett. B 582, 211 (2004) arXiv:hepth/0310227.

[26] H. Boschi-Filho and N. R. F. Braga, "Gauge / string duality and scalar glueball mass ratios," JHEP 0305, 009 (2003) arXiv:hep-th/0212207.

[27] G. F. de Teramond and S. J. Brodsky, "Baryonic states in QCD from gauge / string duality at large N(c)," arXiv:hep-th/0409074.

[28] G. F. de Teramond and S. J. Brodsky, "The hadronic spectrum of a holographic dual of QCD," Phys. Rev. Lett. 94, 201601 (2005) arXiv:hep-th/0501022.

[29] D. K. Hong, T. Inami and H. U. Yee, "Baryons in AdS/QCD," Phys. Lett. B 646, 165 (2007) arXiv:hep-ph/0609270.

[30] J. Erlich, E. Katz, D. T. Son and M. A. Stephanov, "QCD and a holographic model of hadrons," Phys. Rev. Lett. 95, 261602 (2005) arXiv:hep-ph/0501128.

[31] L. Da Rold and A. Pomarol, "Chiral symmetry breaking from five dimensional spaces," Nucl. Phys. B 721, 79 (2005) arXiv:hep-ph/0501218; "The scalar and pseudoscalar sector in a five-dimensional approach to chiral symmetry breaking," JHEP 0601, 157 (2006) arXiv:hep-ph/0510268.

[32] J. Hirn and V. Sanz, "Interpolating between low and high energy QCD via a 5D Yang-Mills model," JHEP 0512, 030 (2005) arXiv:hep-ph/0507049; J. Hirn, N. Rius and V. Sanz, "Geometric approach to condensates in holographic QCD," Phys. Rev. D 73, 085005 (2006) arXiv:hep-ph/0512240].

[33] K. Ghoroku, N. Maru, M. Tachibana and M. Yahiro, "Holographic model for hadrons in deformed AdS(5) background," Phys. Lett. B 633, 602 (2006) arXiv:hep-ph/0510334.

[34] J. Polchinski and M. J. Strassler, "Deep inelastic scattering and gauge/string duality," JHEP 0305, 012 (2003) arXiv:hep-th/0209211. 
[35] R. C. Brower, J. Polchinski, M. J. Strassler and C. I. Tan, "The pomeron and gauge / string duality," arXiv:hep-th/0603115.

[36] J. Babington, J. Erdmenger, N. J. Evans, Z. Guralnik and I. Kirsch, "Chiral symmetry breaking and pions in non-supersymmetric gauge / gravity Phys. Rev. D 69, 066007 (2004) arXiv:hep-th/0306018].

[37] For a recent review of the top-bottom approach to the string/gauge correspondence, including applications to finite temperature and phase transitions, see: K. Peeters and M. Zamaklar, "The string/gauge theory correspondence in QCD," arXiv:0708.1502 [hep-ph] and references therein.

[38] A. Karch, E. Katz, D. T. Son and M. A. Stephanov, "Linear confinement and AdS/QCD," Phys. Rev. D 74, 015005 (2006) arXiv:hep-ph/0602229.

[39] R. R. Metsaev, "IIB supergravity and various aspects of light-cone formalism in AdS space-time," arXiv:hep-th/0002008.

[40] S. J. Brodsky, H. C. Pauli and S. S. Pinsky, "Quantum chromodynamics and other field theories on the light cone," Phys. Rept. 301, 299 (1998) arXiv:hepph/9705477].

[41] D. E. Soper, "The Parton Model And The Bethe-Salpeter Wave Function," Phys. Rev. D 15, 1141 (1977).

[42] S. D. Drell and T. M. Yan, "Connection Of Elastic Electromagnetic Nucleon Form-Factors At Large $\mathrm{Q}^{* *} 2$ And Deep Inelastic Structure Functions Near Threshold," Phys. Rev. Lett. 24, 181 (1970).

[43] G. B. West, "Phenomenological model for the electromagnetic structure of the proton," Phys. Rev. Lett. 24, 1206 (1970).

[44] S. J. Brodsky and S. D. Drell, "The Anomalous Magnetic Moment And Limits On Fermion Substructure," Phys. Rev. D 22, 2236 (1980).

[45] P. Breitenlohner and D. Z. Freedman, "Stability In Gauged Extended Supergravity," Annals Phys. 144, 249 (1982).

[46] L. Infeld, "On a New Treatment of Some Eigenvalue Problems", Phys. Rev. 59, 737 (1941).

[47] S. Eidelman et al. [Particle Data Group], "Review of particle physics," Phys. Lett. B 592, 1 (2004). 
[48] S. J. Brodsky and G. R. Farrar, "Scaling Laws For Large Momentum Transfer Processes," Phys. Rev. D 11, 1309 (1975).

[49] V. A. Matveev, R. M. Muradian and A. N. Tavkhelidze, "Automodellism in the large - angle elastic scattering and structure of hadrons," Lett. Nuovo Cim. 7, 719 (1973).

[50] S. Ferrara, A. F. Grillo and G. Parisi, "Conformal symmetry at lightlike distances and asymptotic behaviour of electromagnetic form factors," Nuovo Cim. A 12, 952 (1972).

[51] R. Baldini, S. Dubnicka, P. Gauzzi, S. Pacetti, E. Pasqualucci and Y. Srivastava, "Nucleon time-like form factors below the N anti-N threshold," Eur. Phys. J. C 11, 709 (1999).

[52] V. Tadevosyan et al. [Jefferson Lab F(pi) Collaboration], "Determination of the pion charge form factor for $Q^{2}=0.60-1.60 \mathrm{GeV}^{2}$," Phys. Rev. C 75, 055205 (2007) arXiv:nucl-ex/0607007.

[53] T. Horn et al. [Fpi2 Collaboration], "Determination of the charged pion form factor at $\mathrm{Q}^{* *} 2=1.60-(\mathrm{GeV} / \mathrm{c})^{* *} 2$ and $2.45-(\mathrm{GeV} / \mathrm{c})^{* *} 2, "$ Phys. Rev. Lett. 97, 192001 (2006) arXiv:nucl-ex/0607005.

[54] H. C. Pauli and S. J. Brodsky, "Discretized Light Cone Quantization: Solution To A Field Theory In One Space One Time Dimensions," Phys. Rev. D 32, 2001 (1985).

[55] A. V. Radyushkin, "Holographic wave functions, meromorphization and counting rules," Phys. Lett. B 642, 459 (2006) arXiv:hep-ph/0605116.

[56] M. E. Peskin and D. V. Schroeder, "An Introduction To Quantum Field Theory," Reading, USA: Addison-Wesley (1995).

[57] H. M. Choi and C. R. Ji, "Conformal Symmetry and Pion Form Factor: Soft and Hard Contributions," Phys. Rev. D 74, 093010 (2006) arXiv:hep-ph/0608148.

[58] E. M. Aitala et al. [E791 Collaboration], "Direct measurement of the pion valence quark momentum distribution, the pion light-cone wave function squared," Phys. Rev. Lett. 86, 4768 (2001) arXiv:hep-ex/0010043.

[59] M. Diehl, "Generalized parton distributions from form factors," Nucl. Phys. Proc. Suppl. 161, 49 (2006) arXiv:hep-ph/0510221. 
[60] S. J. Brodsky and G. P. Lepage, "Exclusive Processes In Quantum Chromodynamics," Adv. Ser. Direct. High Energy Phys. 5, 93 (1989).

[61] D. W. Sivers, S. J. Brodsky and R. Blankenbecler, "Large Transverse Momentum Processes," Phys. Rept. 23, 1 (1976).

[62] C. G. White et al., "Comparison of 20 exclusive reactions at large t," Phys. Rev. D 49, 58 (1994).

[63] R. J. Holt, "Exclusive photonuclear reactions and asymptotic scaling," Phys. Rev. C 41, 2400 (1990).

[64] C. Bochna et al. [E89-012 Collaboration], "Measurements of deuteron photodisintegration up to 4.0-GeV," Phys. Rev. Lett. 81, 4576 (1998) arXiv:nuclex/9808001.

[65] P. Rossi et al. [CLAS Collaboration], "Onset of asymptotic scaling in deuteron photodisintegration," arXiv:hep-ph/0405207.

[66] S. Rock et al., "Measurement of elastic electron - neutron scattering and inelastic electron - deuteron scattering cross-sections at high momentum transfer," Phys. Rev. D 46, 24 (1992).

[67] S. J. Brodsky and B. T. Chertok, "The Asymptotic Form-Factors Of Hadrons And Nuclei And The Continuity Of Particle And Nuclear Dynamics," Phys. Rev. D 14, 3003 (1976).

[68] S. J. Brodsky, C. R. Ji and G. P. Lepage, "Quantum Chromodynamic Predictions For The Deuteron Form-Factor," Phys. Rev. Lett. 51, 83 (1983).

[69] A. Danagoulian et al. [Hall A Collaboration], "Compton scattering cross section on the proton at high momentum transfer," Phys. Rev. Lett. 98, 152001 (2007) arXiv:nucl-ex/0701068.

[70] M. Diehl, T. Feldmann, R. Jakob and P. Kroll, "Linking parton distributions to form factors and Compton scattering," Eur. Phys. J. C 8, 409 (1999) arXiv:hepph/9811253.

[71] A. Chen, "Proton Anti-Proton Pair Production In Two-Photon Collisions At Belle," Photon 2001, Ascona, Switzerland, 2-7 Sep 2001

[72] G. R. Court et al., "Energy Dependence Of Spin Effects In P (Polarized) P (Polarized) $\rightarrow$ PP," Phys. Rev. Lett. 57, 507 (1986). 
[73] I. Mardor et al., "Nuclear transparency in large momentum transfer quasielastic scattering," Phys. Rev. Lett. 81, 5085 (1998).

[74] S. J. Brodsky and G. F. de Teramond, "Spin Correlations, QCD Color Transparency and Heavy Quark Thresholds in Proton Proton Scattering," Phys. Rev. Lett. 60, 1924 (1988).

[75] D. Dutta and H. Gao, "The generalized counting rule and oscillatory scaling," Phys. Rev. C 71, 032201 (2005) [arXiv:hep-ph/0411267].

[76] S. J. Brodsky and A. H. Mueller, "Using Nuclei to Probe Hadronization in QCD," Phys. Lett. B 206, 685 (1988).

[77] G. Bertsch, S. J. Brodsky, A. S. Goldhaber and J. F. Gunion, "Diffractive Excitation In QCD," Phys. Rev. Lett. 47, 297 (1981).

[78] E. M. Aitala et al. [E791 Collaboration], "Observation of color-transparency in diffractive dissociation of pions," Phys. Rev. Lett. 86, 4773 (2001) arXiv:hep$\mathrm{ex} / 0010044$.

[79] B. Clasie et al., "Measurement of Nuclear Transparency for the $A\left(e, e^{\prime} p i^{+}\right)$Reaction," arXiv:0707.1481 [nucl-ex].

[80] A. Airapetian et al. [HERMES Collaboration], "The Q**2-dependence of nuclear transparency for exclusive rho0 production," Phys. Rev. Lett. 90, 052501 (2003) arXiv:hep-ex/0209072. 